\title{
Psychosocial Factors and the Pathogenesis of Chronic Hives: A Survey of Canadian Physicians
}

Moshe Ben-Shoshan ${ }^{1 *}$, Ann Clarke ${ }^{2,3}$ and Amir Raz ${ }^{4}$

${ }^{1}$ Division of Pediatric Allergy and Clinical Immunology, Department of Pediatrics, McGill University Health Center, Montreal, Quebec, Canada ${ }^{2}$ Division of Clinical Epidemiology, Department of Medicine, McGill University Health Center, Montreal, Quebec, Canada

${ }^{3}$ Division of Allergy and Clinical Immunology, Department of Medicine, McGill University Health Center, Montreal, Quebec, Canada

${ }^{4}$ Lady Davis Institute for Medical Research and Jewish General Hospital, Departments of Psychiatry, Neurology and Neurosurgery, and Psychology, McGill University, Montreal, Quebec, Canada

\begin{abstract}
Management of chronic spontaneous urticaria (CSU) is challenging and current drug therapy is often inadequate. Our findings suggest that the majority of Canadian specialists who treat CSU consider psychosocial factors important contributors in its pathogenesis.
\end{abstract}

Controversy currently prevails regarding the role of psychosocial factors in chronic spontaneous urticaria (CSU). Many clinicians intimate that psychosocial parameters may contribute, albeit marginally, to the chain of events leading to CSU. However, informal and personal communications, from at least some prominent practitioners, express the opinion that such factors have little, if any, clinical effect on the development of CSU. This discrepancy is surprising in light of several independent reports documenting an association between CSU and psychosocial factors such as obsessive-compulsive disorder (The most common Axis I disorder in CSU patients was obsessive-compulsive disorder ( diagnosed in $25.8 \%$ of these patients)) [1], anxiety (compared to the control group, individuals with CSU had significantly higher scores on anxiety scales) [2], insomnia (e.g. in a study on 75 consecutive cases with CSU and 133 controls with tinea pedis, patients with CSU had significantly more severe insomnia) [3] and acute life-changing events ( $16 \%$ of individuals with CSU had stressful life events - e.g. death of a loved one within 1 year preceding onset or exacerbation) [4]. Recent research findings reveal, furthermore, numerous ways in which the nervous and immune systems interact, including through activation of the Hypothalamic-Pituitary axis leading to mast cell activation and CSU [5].

The objectives of this preliminary investigation were to probe the opinions of Canadian physicians regarding thelink between psychosocial factors and the pathogenesis of CSU. Using an open source web-based application tool, we enlisted the services of a behavioral scientist with expertise in designing computerized questionnaires to create an expressly tailored, invalidated, survey de novo (see www.tinyurl.com/ CSUpsych). Having circulated a single email to all 153 members from the directory of Canadian allergy and immunology specialists, we received 114 anonymous replies, yielding a response rate of $74.50 \%$. Approved by the appropriate ethics committee at McGill University, our 1-minute survey collected basic demographic information and an indication of whether respondents felt that psychological factors played a role in the pathogenesis of CSU. Affirmative responses led respondents to rank the relative contribution for several parameters on a 5-point

\begin{tabular}{|l|c|c|c|c|c|}
\hline & $\begin{array}{c}1 \\
\text { (not at all) }\end{array}$ & 2 & 3 & 4 & $\begin{array}{c}5 \\
\text { (Extremely) }\end{array}$ \\
\hline Stress & & & & & \\
\hline Anxiety & & & & & \\
\hline $\begin{array}{l}\text { Depression } \\
\text { Insomnia }\end{array}$ & & & & & \\
\hline Psychopathology & & & & & \\
\hline Life events & & & & & \\
\hline
\end{tabular}

Figure 1A: Likert scale for the different factors.
Likert scale (Figures 1A and B).Our results show that $78.07 \%$ (95\%CI, $69.15 \%-85.05 \%$ ) of respondents believe that psychosocial factors play a role in the pathogenesis of CSU. Stress, anxiety, depression, insomnia, psychopathology and life events were assigned high likelihood - values of $4-5$ in $71.91 \%(61.23 \%, 80.68 \%), 66.29 \%$ (95\% CI, $55.40 \%, 75.76 \%)$, $29.21 \%$ (95\% CI, $20.30 \%, 39.95 \%$ ), $25.84 \%$ (95\% CI, $17.40 \%, 36.40 \%$ ), $17.98 \%$ (95\% CI, $10.93 \%, 27.85 \%$ ), and $58.43 \%$ (95\% CI, $47.49 \%$, $68.63 \%)$ of respondents, respectively. Our results indicate that most allergists think that stress, anxiety and life events play a major role in the pathogenesis of CSU. In contrast, there is more controversy regarding the role of depression, insomnia and psychopathology (Figure 1B). It is possible that clinicians deem depression, insomnia and psychopathology as consequences rather than triggers of CSU and hence assign them a lower rank in the pathogenesis of CSU.

Methodological advantages of web-based surveys include expediency-allowing for efficient data collection and timely results; casting a wide net while reducing the cost relative to the sample size; and providing respondents with a guarantee of anonymity. Shortcomings of web-based surveys include the exclusion of individuals without internet access thereby introducing coverage error-hardly a problem for the population contacted herein. Finally, in light of the robust response rate and the nature of this survey, the general methodological concern of non-response error-applicable to any survey-is unlikely to undermine the present results. Whereas the present findings draw on a subgroup representing the constituency of allergists, it is probable that our results reflect clinical undercurrents that may be of general interest as allergists care for the vast majority of individuals with CSU in Canada (e.g., allergists and dermatologists see 13 and 4 individuals with CSU per month, respectively) [6]. Given that clinical experience shapes

*Corresponding author: Moshe Ben-Shoshan, Assistant Professor, Division of Pediatric Allergy and Clinical Immunology, Department of Pediatrics, McGill University Health Center, Montreal, Quebec, Canada; E-mail: moshebenshoshan@gmail.com

Received December 17, 2011; Accepted February 22, 2012; Published February 27, 2012

Citation: Ben-Shoshan M, Clarke A, Raz A (2012) Psychosocial Factors and the Pathogenesis of Chronic Hives:A Survey of Canadian Physicians. J Aller Ther 3:113. doi:10.4172/2155-6121.1000113

Copyright: (c) 2012 Ben-Shoshan M, et al. This is an open-access article distributed under the terms of the Creative Commons Attribution License, which permits unrestricted use, distribution, and reproduction in any medium, provided the original author and source are credited. 
Citation: Ben-Shoshan M, Clarke A, Raz A (2012) Psychosocial Factors and the Pathogenesis of Chronic Hives:A Survey of Canadian Physicians. J

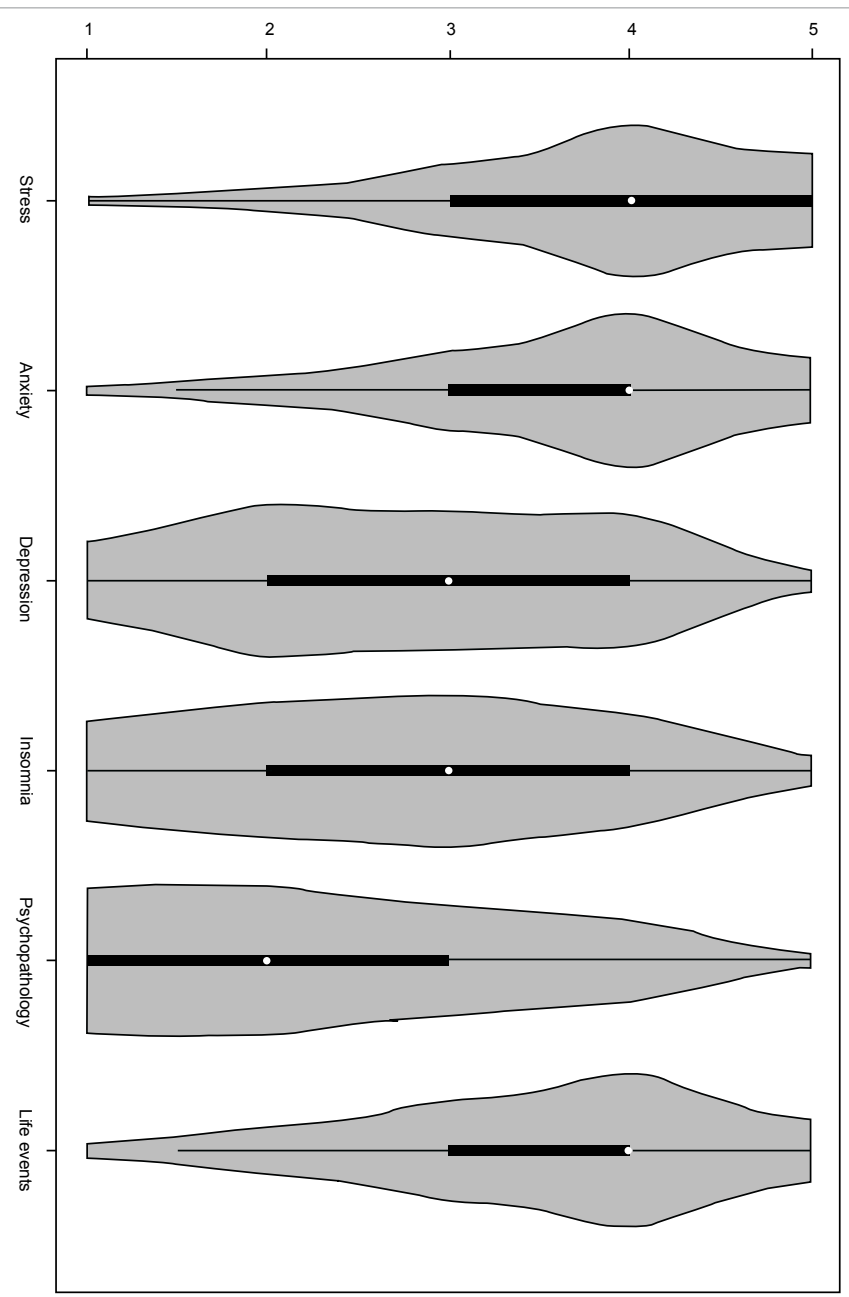

Figure 1B: Distribution of data per factor: Vertical box plots show density traces. Circles and bold lines represent medians and interquantile ranges, respectively. Responses for Depression, Insomnia, and Psychopathology follow near-normal distributions whereas results for the higher-impact values associated with Stress, Anxiety, and Life-changing Events indicate an endorsement of the involvement of these psychosocial parameters in the origination and development of CSU. the opinion of medical practitioners [7] and that published studies report associations between psychopathological factors and CSU[15], a behavioral approach may arguably contribute to the clinical management of CSU. However, the only way to ascertain the validity of such a strategy is to conduct randomized controlled trials comparing a behavioral approach with mainstream medicine in patients with CSU.

\section{Acknowledgement}

The authors thank Ms. Irina Demacheva, Ms. Maggie Latucca, and the Educational Technologies team at McGill University for data management and technical support associated with the online survey. In addition, Dr. Raz acknowledges the kind support of the Canada Research Chair program as well as grant support from the Canadian Institute of Health Research, the Natural Sciences and Engineering Research Council of Canada, and the Oxford-McGill Neuroscience Collaboration; Dr. Ben-Shoshan acknowledges the support of Allergy, Genes, and Environment (AllerGen) Network of Centres of Excellence.

\section{References}

1. Uguz F, Engin B, Yilmaz E (2008) Axis I and Axis II diagnoses in patients with chronic idiopathic urticaria. J Psychosom Res 64: 225-229.

2. Sperber J, Shaw J, Bruce S (1989) Psychological components and the role of adjunct interventions in chronic idiopathic urticaria. Psychother Psychosom 51: 135-141.

3. Yang HY, Sun CC, Wu YC, Wang JD (2005) Stress, insomnia, and chronic idiopathic urticaria--a case-control study. J Formos Med Assoc 104: 254-263.

4. Malhotra SK, Mehta V (2008) Role of stressful life events in induction or exacerbation of psoriasis and chronic urticaria. Indian J Dermatol Venereol Leprol 74: 594-599.

5. Broom BC (2010) A reappraisal of the role of 'mindbody' factors in chronic urticaria. Postgrad Med J 86: 365-370.

6. Sharma JK, Miller R, Murray S (2000) Chronic urticaria: a Canadian perspective on patterns and practical management strategies. J Cutan Med Surg 4: 89-93.

7. Merry AF (2011) To do or not to do?--How people make decisions. J Extra Corpor Technol 43: 39-43. 